\title{
Assessment of fetal development by HRV and chaotic global techniques
}

\author{
David M. Garner ${ }^{1}$, Peter van Leeuwen², Dietrich Grönemeyer ${ }^{3}$, Shakeeb Moosavi ${ }^{4}$
}

DOI: http://dx.doi.org/10.7322/jhgd.119258

\begin{abstract}
Introduction: Fetal heart rate and its variability during the course of gestation have been extensively researched. The overall reduction in heart rate and increase in fetal HRV is associated with fetal growth and the increase in neural integration. The increased complexity of the demands on the cardiovascular system leads to more variation in the temporal course of the heart rate which has been shown to be reflected in measures of complexity. The aim of this work was to investigate novel complexity measures with respect to their ability to quantify changes over gestational age in individual fetuses consistently and in a stable manner.
\end{abstract}

Methods: We examined 215 fetal magnetocardiograms (FMCG), each of 5 min duration, in 11 fetuses during the second and third trimesters (at least 10 data sets per fetus). From the FMCG we determined the fetal RR beat durations. For each 5 min time-series of RR intervals we then calculated Shannon entropy, high spectral entropy, high spectral Detrended Fluctuation Analysis, spectral Multi-Taper Method as well as the standard deviation and two commonly used complexity measures: Approximate Entropy and Sample Entropy. For each subject and HRV measure, we performed regression analysis with respect to gestational age. The coefficient of determination $\mathrm{R}^{2}$ was used to estimate 'goodness-of-fit', the slope of the regression indicated the strength of the individual dependency on gestational age.

Results: We found that the new complexity measures do not outperform ApEn.

Conclusion: This study has now rejected the hypothesis that the spectral complexity measures outperform those applied previously.

Key words: entropy, heart rate variability, complexity, chaos, power spectra.

\section{INTRODUCTION}

The change in fetal heart rate and its variability during the course of gestation has been extensively researched ${ }^{1-4}$. Increases in fetal Heart Rate Variability (HRV) are associated with fetal growth and are considered to reflect the increase in neural integration as the fetus develops. Recent studies have demonstrated that these changes are related to adjustment of activity in the sympathetic and parasympathetic branches of the autonomic nervous system ${ }^{5,6}$. The development of autonomic inputs on the cardiovascular system permits an increasingly differentiated interaction within organ systems. It has been observed that specific fetal activity patterns emerge during gestation. As early as the beginning of the third trimester, different heart rate patterns can be identified $^{7}$. The various patterns are also associated with different effects on fetal $\mathrm{HRV}^{8,9}$.
In a previous study, some of us ${ }^{1}$ showed that, beyond known factors such as gestational age or behavioural state, an intra-individual effect on fetal HRV may also be present. In particular, we found that Approximate Entropy (ApEn), a complexity measure, captured individual aspects of HRV well, better than the linear measures we studied. This was the motivation to examine other nonlinear measures in this context. On the one hand, ApEn is very dependent on parameter choice, i.e. dimension $\mathrm{m}$ and radius $\mathrm{r}^{10}$. Although $\mathrm{m}$ (length of sequences) and $\mathrm{r}$ (tolerance for accepting matches) are critical in the values of ApEn and SampEn obtained, there is no guideline available to optimize their values. There is considerable skill and technique to citing the correct (optimal) values.

We term three of these new spectral measures "chaotic globals" - high spectral entropy (hsEntropy)", high spectral Detrended Fluctuation Analysis $(h s \text { DFA })^{11}$ and spectral Multi-Taper Method (sMTM) ${ }^{12}$. They are

\footnotetext{
1 Oxford Brookes University, Gipsy Lane, Oxford OX3 OBP, United Kingdom.

2 University Witten/Herdecke, Universitätsstr. 142, D - 44799 Bochum, Germany.

3 University Witten/Herdecke, Universitätsstr. 142, D - 44799 Bochum, Germany.

4 Faculty of Health and Life Sciences, Oxford Brookes University, Gipsy Lane, Oxford OX3 OBP, United Kingdom.

Corresponding author: David M. Garner - E-mail: dgarner@brookes.ac.uk
}

Suggested citation: Garner DM, Leeuwen PV, Grönemeyer D, Moosavi S. Assessment of fetal development by HRV and chaotic global techniques. J Hum Growth Dev. 26(1): 162-173. Doi: http://dx.doi.org/10.7322/jhgd.119258.

Manuscript submitted Jun 10 2016, accepted for publication Jun 262016. 
"global" since founded on the application of the power spectrum to the whole dataset. This has a number of benefits which could be useful here in fetal HRV. For instance, they do not need long datasets such as with previous measures of complexity such as Shannon Entropy ${ }^{13}$ and Detrended Fluctuation Analysis (DFA) ${ }^{14}$. This is because more of the information is used in generating these parameters response; only the phase information is lost. This has been proven in previous studies which have investigated the effect of flexible-pole exercise ${ }^{15}$, diabetes ${ }^{16}$, Chronic Obstructive Pulmonary Disease ${ }^{17}$ on these chaotic globals. Standard measures of chaos were outperformed by the chaotic globals and only relied on 256 to 1000 RR beat durations to discriminate between the experimental and control datasets.

A potential pitfall of chaotic globals is that they may not be as sensitive to the data over time as are the time-series methods. There is a trade-off between the nonlinear frequency responsiveness of the spectral techniques; and the sensitivity of the traditional techniques. It is proposed that the lack of sensitivity over time is due to the fact that there is heavy normalisation in the generation of chaotic globals. This normalisation flattens the response of the parameters. Also, spectral measures based on power spectra have less data points to assess than with time-series methods. Therefore, these spectral techniques may not be as receptive over the gestational age for fetal HRV but may have the potential to outperform standard nonlinear methods.

It is thus the aim of this study to complement the results of the previous study by re-examining the data using further complexity parameters. Additionally to ApEn we examine hsEntropy, hsDFA, sMTM, Shannon Entropy, Sample Entropy (SampEn) ${ }^{18}$ and the Standard Deviation (SD). Here, the time-series are for 5 minutes, as recommended by the Task Force of the European Society of Cardiology and the North American Society of Pacing and Electrophysiology ${ }^{19}$. Our hypothesis was that the new spectral measures of complexity would outperform the ApEn, or the similar technique SampEn, with regard to correlating with gestational age. In addition we assessed whether fetal HRV has an individual aspect.

\section{METHODS}

\section{Data Acquisition}

The data used in this study had been collected in previous studies examining fetal $\operatorname{HRV}(1,2)$. These studies were approved by the local ethics committee. All mothers, aged 27-39 years, gave written informed consent after verbal and written explanation of the aim of the study and the procedures used.

All mothers were healthy with no history of endocrine, respiratory or cardiovascular disorders. The data sets were selected such that each fetus included had a minimum of ten sets collected over at least ten weeks. Eleven fetuses with a total of 215 heartbeat data sets fulfilled these criteria.
The data acquisition was from the 16 th to 42 nd week. For the period between the 19th and 39th week, there were overall at least six data sets available per week of gestation. The children were born on or after the 40th week of gestation with one exception in the 36 th week. The maximal gestational interval throughout was 42 weeks. All were born healthy with birth weights ranging from 2.68 to 4.18 kilos

Fetal magnetocardiogram (FMCG) data were acquired noninvasively using one of two biomagnetometer sensor systems: either a 37-channel Krenikon (Siemens, Erlangen) or a 67-channel Magnes 1300C (4D Neuroimaging, San Diego). After disposing of any metal objects that might cause signal irregularities, the mothers lay in a comfortable supine position on the patient table. The sensor was placed approximately $1 \mathrm{~cm}$ above the abdomen ensuring that fetal signals were available in a number of channels. Data were sampled for 5-10 min at $1 \mathrm{kHz}$ using a band pass of 1-200 Hz. In order to minimize the effect of external noise, all acquisitions were performed in a standard 3-layer shielded room (Vakuumschmelze AK3b, Erlangen).

\section{Pre-processing: selection of time series data}

In order to obtain a time-series of the fetal RR interval variability from each data set, the signals were acquired at each recording session using the sampling parameters described above. After noise reduction using software gradiometers, all maternal beats were identified using an appropriate maternal QRS template. A signal-averaged maternal PQRST segment was then digitally subtracted at each beat, resulting in a signal largely free of maternal influence. In this signal, all fetal beats were identified to an accuracy of 1 millisecond using an appropriate fetal QRS template in a channel with a high signal-to-noise ratio. Falsely-marked signal abnormalities were manually removed and missed beats were manually added. In each data set, the resulting $\mathrm{R}$-times were used to determine the RR intervals. The first $600 \mathrm{RR}$ intervals, were used in the datasets to be assessed by the six parameters. This amounted to $3.5-5.0$ minutes of data depending on heart rate.

On the basis of time-series of exactly 600 RR beat durations; the Standard Deviation of the RR beat durations was calculated. Furthermore, six complexity measures were determined: Shannon entropy, high spectral Entropy, hsDFA, sMTM, SampEn and ApEn. For the fetal HRV measures we performed regression analysis with respect to gestational age, calculating the slope and y-intercept of the regression as well as the coefficient of determination $\mathrm{R}^{2}$ as a measure of 'goodness-of-fit.' This was done for all the data sets combined as well as for the data of each of the 11 individual subjects.

\section{Complexity Statistics}

\section{Shannon Entropy:}

Shannon entropy ${ }^{20,21}$ is defined in relation to information theory. A low entropy dataset is highly predictable 
- whereas a high entropy dataset is less predictable and more disordered. Conversion of macroscopic thermodynamic parameters into microscopic molecular entities is complex. Statistical entropy functions cannot be easily applied or interpreted for living systems. Study in this area has been motivated by analysis of time-series derived from statistical physics ${ }^{22}$.

\section{High Spectral Techniques}

A potential criticism in previous studies on diabetes $^{16}$ and childhood obesity ${ }^{23}$ with respect to chaotic global parameters is that the spectral entropy and spectral Detrended Fluctuation Analysis (sDFA) analysis may be more sensitive if we applied the Shannon entropy and
DFA algorithms to the multi-taper method (MTM) spectrum $^{24}$; rather than the Welch power spectrum ${ }^{25}$. Thus the spectra applied in all three chaotic global parameters would correspond.

$\mathrm{MTM}^{24,26}$ is useful for spectral estimation and signal reconstruction, of a time-series of a spectrum that may contain broadband and line components. It is non-parametric since it does not apply an a priori, parameter dependent model of the process that generated the time-series under analysis. In addition, MTM reduces the variances of spectral estimates by using a small set of tapers. Data is pre-multiplied by orthogonal tapers created to minimize the spectral leakage owing to the finite length of the time-series. A set of independent approximations of the power spectrum is calculated. Functions identified as discrete prolate spheroidal se-

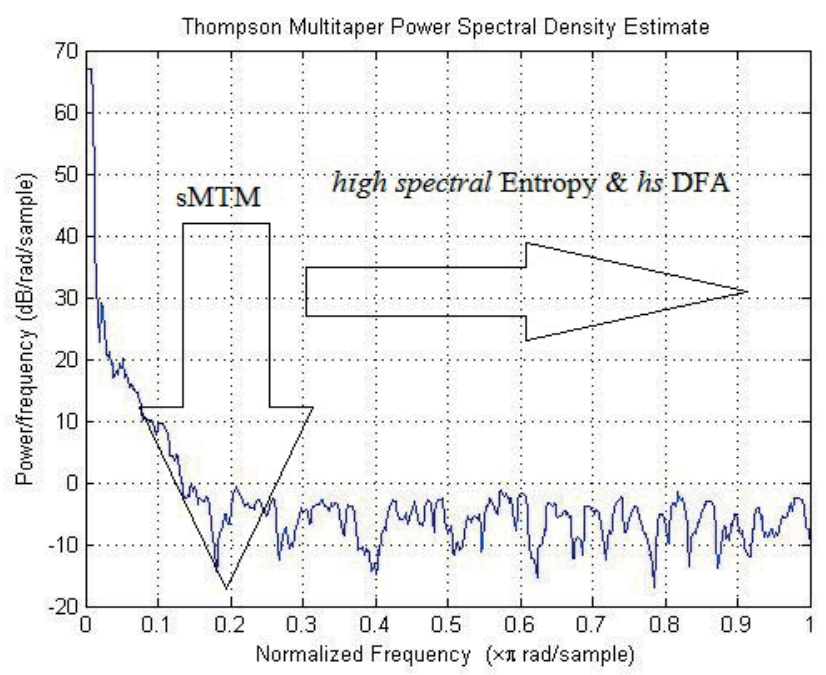

Figure 1: A multi-taper method (MTM) power spectrum of a time-series of RR fetal beat durations. SMTM is the area beneath the spectrum; yet above the baseline created by broadband noise as the signal becomes chaotic. hsEntropy and hsDFA are derived by applying the standard Shannon Entropy and Detrended Fluctuation Analysis (DFA) functions to the MTM power spectrum. Throughout this study the parameters for MTM are always set at the following: (i) sampling frequency of $1 \mathrm{~Hz}$; (ii) time bandwidth for the discrete prolate spheroidal sequences (DPSS) is 3; (iii) FFT length of 256; (iv) Thomson's adaptive nonlinear combination method to combine individual spectral estimates.

quences (DPSS) ${ }^{27}$ are a set of functions which optimize the tapers. They are defined as eigenvectors of a Rayleigh-Ritz minimization problem ${ }^{28}$. For settings of the MTM power spectrum, see the legend for Figure 1 above.

\section{High Spectral Entropy (hsEntropy):}

Spectral entropy ${ }^{29}$ is a function of Shannon entropy ${ }^{13}$ where the horizontal axis is frequency and the vertical axis is amplitude in the two-dimensional sense. hsEntropy is also a function of the irregularity of amplitude and frequency of the power spectrums peaks. This novel function is however derived by applying Shannon entropy to the MTM power spectrum. This output is measured in arbitrary units. We calculate an intermediate parameter which is the median Shannon entropy of the value obtained from three different power spectra using the MTM power spectra under three test conditions: a perfect sine wave, uniformly distributed random variables, and finally the experimental oscillating signal. These values are then normalized mathematically so that the sine wave gives a value of zero, uniformly random variables give unity, and the experimental signal between zero and unity. This final value corresponds to hsEntropy. A further advantage here is that the only information lost in the analysis are the phase differences. Moreover, noise is not problematic as it is not over-emphasized as would be the case if applied in the conventional manner to interpeak temporal separations.

\section{High Spectral Detrended Fluctuation Analysis (hsDFA):}

DFA $^{14,30}$ can be applied to datasets where statistics such as mean, variance and autocorrelation vary with time. The difference with the sDFA algorithm is that the DFA is applied to the frequency rather than time on the 
horizontal axis. Regarding DFA according to Donald$\operatorname{son}^{31}$ the time-series of length $\mathrm{k}$ was integrated as follows.

$$
y(k)=\sum_{i=1}^{k}(x(i)-\bar{x})
$$

The integrated time-series was then divided into equally sized and non overlapping windows of length w. A linear regression line was fitted through the data in each window and the time-series locally detrended by subtracting the regression line from the data. The root mean square fluctuation $\mathrm{F}(\mathrm{w})$ of the integrated and detrended time-series was then used to calculate values of $\mathrm{w}$.

$$
F(w)=\left[\frac{1}{N} \sum_{k=1}^{N}\left[y(k)-y_{w}(k)\right]^{2}\right]^{\frac{1}{2}}
$$

The scaling exponent obtained as the slope of the straight line fit to $\mathrm{F}(\mathrm{w})$ against $\mathrm{w}$ on a log-log plot as:

$$
F(w) \propto w^{\alpha} .
$$

To obtain hsDFA we calculate the spectral adaptation in exactly the same way as for hsEntropy using a MTM power spectrum with the same settings; but DFA rather than Shannon entropy is the algorithm imposed. hsDFA is subtracted from unity, (1- hsDFA); to make it comparable, as it responds in the opposite manner to increasing chaos to the other parameters.

\section{Spectral Multi-Taper Method (sMTM)}

sMTM is founded on the increased intensity of broadband noise in power spectra generated by irregular and chaotic signals. It is an exclusively spectral technique. The main features of MTM are: (1) Efficient in detecting periodic components; (2) A random signal may generate many false peaks which may or may not be significant; (3) There are two ways of testing the spectrum (red-noise ${ }^{32}$ and harmonic tests). We set the parameters for MTM at the same as those for hsEntropy and hsDFA. For the choice of weightings and other details it is advisable to consult Percival and Walden ${ }^{24}$ or Thomson ${ }^{33}$. sMTM is the area between the MTM power spectrum and the baseline.

\section{Approximate Entropy}

$\mathrm{ApEn}^{10}$ is a procedure used to assess the level of regularity and the unpredictability of changes over time-series. The creation of ApEn was motivated by the search for a distribution free measure of these fluctuations. ApEn is not predicated on the underlying distribution of the data as with Shannon Entropy. Instead it relies on sequence recurrences and therefore it is more suitable to shorter length signals. It also makes the use of model estimation invalid removing the liability for false estimation based on poor model selection. It was developed as a tool that could be applied to both correlated random and noisy deterministic processes. Its motivation originates from nonlinear dynamics, complexity theory; especially the entropy being approximated is Kolmogorov-Sinai Entropy $^{34}$. This is generally finite for deterministic processes and infinite for random processes. ApEn is the logarithmic ratio of component wise matching sequences from the signal length, N. Other parameters to be specified include $r$ which we set to 4 . The factor $m$, is the run length of data to be compared; which we set to 2 . It is measured as an integer count of discrete time bins. A minimum value of zero for ApEn would indicate a totally predictable series. ApEn is described algorithmically as follows ${ }^{35}$.

Given $\mathrm{N}$ data points from a time-series $\{\mathrm{x}(\mathrm{n})\}=$ $x(1), x(2), \ldots . x(N)$, one should follow these steps to compute ApEn:

1. Form $\mathrm{N}-\mathrm{m}+1$ vectors $\mathrm{X}(1), \mathrm{X}(2), \ldots, \mathrm{X}(\mathrm{N}-\mathrm{m}+$ 1) defined by $X(i)=[x(i), x(i+1), \ldots, x(i+m-1)], i=1, \ldots$, $\mathrm{N}-\mathrm{m}+1$.

These vectors represent $\mathrm{m}$ consecutive $\mathrm{x}$ values, beginning with the $\mathrm{i}^{\text {th }}$ point.

2. Define the distances between $X(i)$ and $X(j), d[X-$ (i), $\mathrm{X}(\mathrm{j})]$, as the maximum norm,

$$
d[X(i), X(j)]=\max _{k=1, \ldots, m}(|x(i+k-1)-x(j+k-1)|)
$$

3. For a given $X(i)$, count the number of $j(j=1, \ldots$, $\mathrm{N}-\mathrm{m}+1)$, so that $\mathrm{d}[\mathrm{X}(\mathrm{i}), \mathrm{X}(\mathrm{j})] \leq r$, denoted as $N^{m}(i)$, then, for $\mathrm{i}=1, \ldots \mathrm{N}-\mathrm{m}+1$.

$$
C_{r}^{m}(i)=\frac{N^{m}(i)}{N-m+1} \text { with a tolerance } r \text {, the fre- }
$$

quency of patterns similar to a given one of window length $m$.

4. Compute the natural logarithm of each $C_{r}^{m}(i)$ and average it over i,

$$
\phi^{m}(r)=\frac{1}{N-m+1} \sum_{i=1}^{N-m+1} \ln C_{r}^{m}(i)
$$

5. Increase the dimension to $\mathrm{m}+1$. Repeat steps 1-4 and find $C_{r}^{m+1}(i)$ and $\phi^{m+1}(r)$

6. ApEn is defined as

$$
\operatorname{ApEn}(m, r, N)=\phi^{m}(r)-\phi^{m+1}(r)
$$

The advantages of ApEn include a low computer processor demand; due to the fact that it can adapt to small sample lengths. As a consequence, it can be applied online and retrospectively in the clinical situation or in the research laboratory. It is also robust in the presence of significant noise. A disadvantage of the technique is that it is very dependent on the parameters choice. This makes the results from ApEn especially difficult to interpret and is therefore only best consulted in combination with other methods, which then helps understand the datasets response. Other methods which are suitable to make comparisons with include SampEn; where the same parameters would be set for tolerance, $r$ and run length, $\mathrm{m}$ - discussed above.

\section{Sample Entropy}

The ApEn algorithm counts each sequence as matching itself to avoid the occurance of $\ln (0)$ in the calculations which introduces bias. SampEn ${ }^{36-38}$ aims reduce the bias. A run length $\mathrm{m}$ and a tolerance window $\mathrm{r}$, must 
be specified to compute SampEn. SampEn (m, r, N) is the negative logarithm of the conditional probability that two sequences similar to $\mathrm{m}$ points remain similar at the next point, where self-matches are not included in calculating the probability. A lower value of SampEn indicates more self-similarity in the time-series. It is highly independent of dataset length and is more statistically robust than ApEn. The algorithm is simpler and eliminates self matches. The algorithm for SampEn is discussed mathematically ${ }^{35}$ :

1. Given $N$ data points from a time-series $\{x(n)\}=$ $x(1), x(2), \ldots x(N)$.

2. Form $m$ - vectors $X_{\mathrm{m}}(1), X_{\mathrm{m}}(2) \ldots X_{\mathrm{m}}(N-m+1)$; the distance between $X_{\mathrm{m}}(i)$ and $X_{\mathrm{m}}(j)$ is defined as:

$$
d[X(i), X(j)]=\max _{k=1, \ldots, m}(|x(i+k-1)-x(j+k-1)|)
$$

3 . For a given $X_{\mathrm{m}}(i)$, count the number of $j(1 \leq j \leq$ $N-m, j \neq i)$ denoted as $B_{i}$, such that the distance between $X_{\mathrm{m}}(i)$ and $X_{\mathrm{m}}(j)$ is less than or equal to $r$.

4. Then, $1 \leq i \leq N-m, B_{i}^{m}(r)=\frac{1}{N-m-1} B_{i}$, with,

$$
B^{m}(r)=\frac{1}{N-m} \sum_{i=1}^{N-m} B_{i}^{m}(r),
$$

5. We increase the dimension to $m+1$ and calculate $A_{i}$ as the number of $X_{\mathrm{m}+I}(i)$ within $r$ of $X_{\mathrm{m}+l}(j), j$ is from 1 to $N-m(j \neq i)$.

6. We then define $A_{i}^{m}(r)=\frac{1}{N-m-1} A_{i}$

7. Where $A^{m}(r)=\frac{1}{N-m} \sum_{i=1}^{N-m} A_{i}^{m}(r)$
8. $B^{m}(r)$ is the probability that two sequences will match for $m$ points

9. $A^{m}(r)$ is the probability that two sequences will match for $m+1$ points

$$
\text { 10. } \operatorname{SampEn}(m, r, N)=-\ln \left[\frac{A^{m}(r)}{B^{m}(r)}\right]
$$

It is important to consider ApEn and SampEn together as families of metrics. Comparisions with fixed $\mathrm{m}$, $\mathrm{r}$, and $\mathrm{N}$. N is the length of the time sequences to be compared whereas $r$ is the tolerance for accepting matches. As with ApEn in this study $r$ we set to 4 . The factor $m$, is the length of sequences compared which we set to a window of 2 .

\section{Correlation - Least Squares Method}

Once the HRV measures have been calculated using the described algorithms above, they are plotted versus gestational age in weeks. The linear regressions were achieved by the least squares method. From the linear regression the $\mathrm{R}^{2}$ values, the slopes and the $\mathrm{y}$-intercepts are derived.

\section{RESULTS}

The individual linear regressions of fetal HRV measures against gestational age for each method are shown for one typical individual fetus in Figure 2. The mean 'goodness-of-fit' (R2) values for each method are compared in Table 1 and Figure 3. Similarly, the mean values for slope of the linear regressions is provided in Table 2 (Figure 4) and the mean y-intercept for the linear regressions is provided in Table 3 (Figure 5).

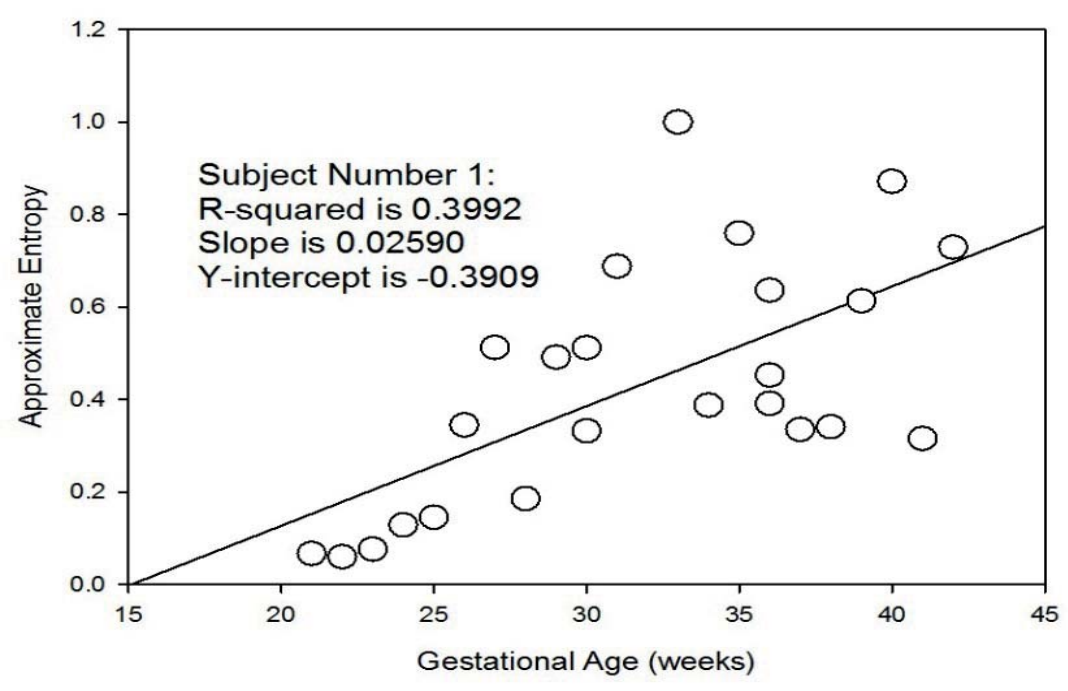

Figure 2: Subject Number 1: The linear regression for Approximate Entropy of fetal HRV versus gestational age in weeks. The linear regressions were achieved by the least squares method. From the linear regression the $\mathrm{R}^{2}$ values, the slopes and the $\mathrm{y}$-intercepts are derived. 


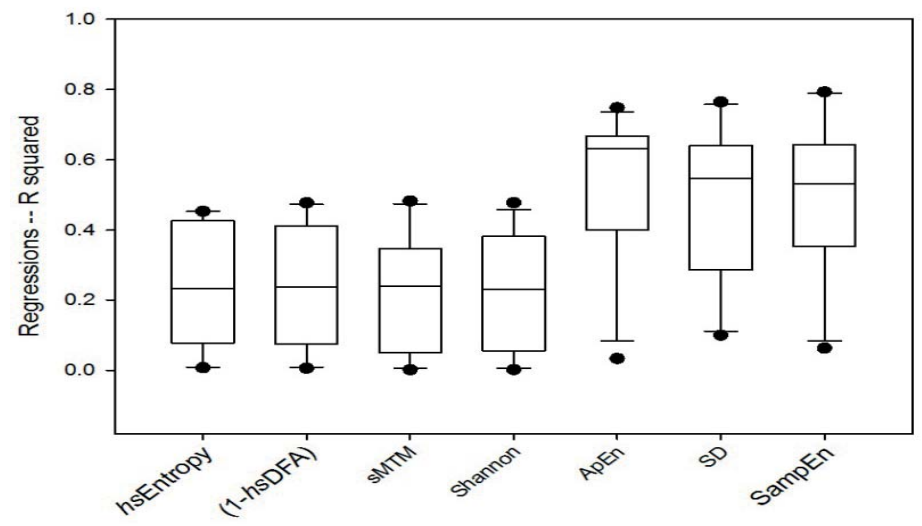

Figure 3: 'Goodness-of-fit' of the seven HRV measures with respect to the gestational age. The box plot illustrates the individual $\mathrm{R}^{2}$ values for each of the HRV measures. As hsDFA decreases with increasing irregularity, the parameter is subtracted from unity (1-hsDFA). The point closest to the zero is the $5^{\text {th }}$ percentile and the point farthest away is the $95^{\text {th }}$ percentile. The boundary of the box closest to zero indicates the $25^{\text {th }}$ percentile, a line within the box marks the median, and the boundary of the box farthest from zero indicates the $75^{\text {th }}$ percentile. Whiskers (or error bars) above and below the box indicate the $90^{\text {th }}$ and $10^{\text {th }}$ percentiles.

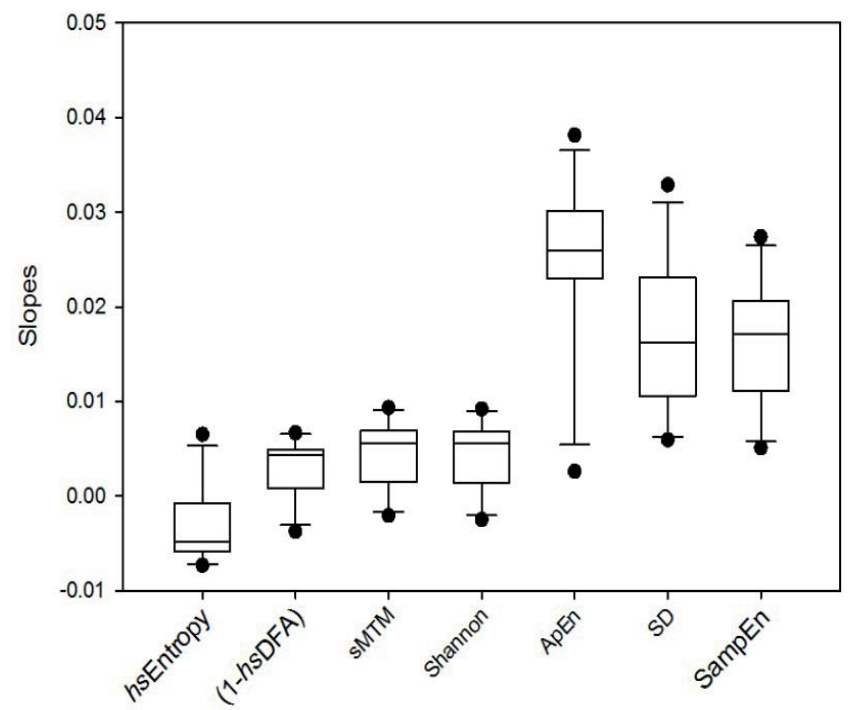

HRV Measures

Figure 4: The box plot above illustrates the individual slope values for each of the HRV measures versus gestational age. As $h s D F A$ decreases with increasing irregularity, the parameter is subtracted from unity (1-hsDFA).

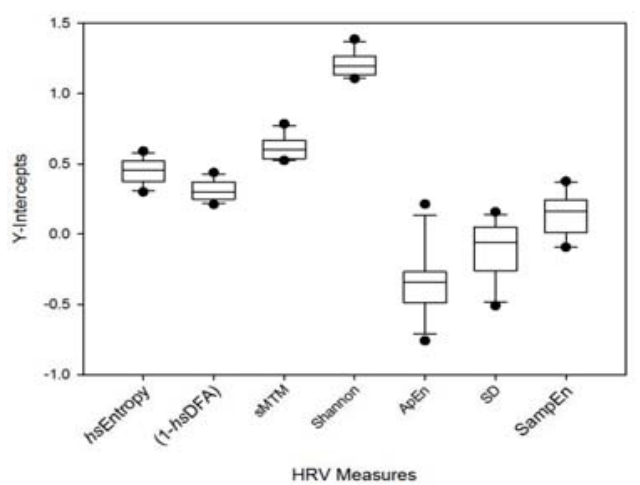

Figure 5: The box plot above illustrates the individual y-intercepts for each of the seven HRV measures versus gestational age. As hsDFA decreases with increasing irregularity, the parameter is subtracted from unity (1-hsDFA). 
Table 1: $\mathrm{R}^{2}$ values (Linear Regression) - Seven parameters versus gestational age for each individual fetus and then, for the complete dataset; where the number of recordings was $N=215$. The parameters were applied to the RR beat durations

\begin{tabular}{cccccccc}
$\begin{array}{c}\text { Subject } \\
\text { Number }\end{array}$ & hsEntropy & (1-hsDFA) & $\begin{array}{c}\text { Spectral } \\
\text { MTM }\end{array}$ & $\begin{array}{c}\text { Shannon } \\
\text { Entropy }\end{array}$ & ApEn & $\begin{array}{c}\text { Standard } \\
\text { Deviation }\end{array}$ & $\begin{array}{c}\text { Sample } \\
\text { Entropy }\end{array}$ \\
$\mathbf{1}$ & 0.2334 & 0.2364 & 0.2479 & 0.2458 & 0.3992 & 0.1585 & 0.3539 \\
$\mathbf{2}$ & 0.0073 & 0.0062 & 0.0020 & 0.0020 & 0.0338 & 0.0999 & 0.0630 \\
$\mathbf{3}$ & 0.0774 & 0.1062 & 0.1539 & 0.1446 & 0.5226 & 0.5618 & 0.5805 \\
$\mathbf{4}$ & 0.2504 & 0.2511 & 0.2404 & 0.2297 & 0.6667 & 0.5472 & 0.5447 \\
$\mathbf{5}$ & 0.0150 & 0.0204 & 0.0240 & 0.0210 & 0.6490 & 0.2858 & 0.1672 \\
$\mathbf{6}$ & 0.4533 & 0.4773 & 0.4825 & 0.4775 & 0.7480 & 0.3320 & 0.4612 \\
$\mathbf{7}$ & 0.4502 & 0.4553 & 0.4426 & 0.3820 & 0.4243 & 0.7288 & 0.6427 \\
$\mathbf{8}$ & 0.0825 & 0.0753 & 0.0516 & 0.0558 & 0.2879 & 0.3097 & 0.5321 \\
$\mathbf{9}$ & 0.3340 & 0.3438 & 0.3179 & 0.3162 & 0.6608 & 0.6409 & 0.7928 \\
$\mathbf{1 0}$ & 0.4270 & 0.4110 & 0.3463 & 0.3836 & 0.6882 & 0.7644 & 0.7749 \\
$\mathbf{1 1}$ & 0.1313 & 0.1625 & 0.2098 & 0.1922 & 0.6305 & 0.5834 & 0.5247 \\
$\mathbf{A l l}$ & 0.0127 & 0.0253 & 0.0526 & 0.0458 & 0.4750 & 0.2705 & 0.3047 \\
\hline
\end{tabular}

Table 2: Slopes (Linear Regression) - Seven parameters versus gestational age for each individual fetus and then, for the complete dataset. The total number of recordings was $\mathrm{N}=215$; calculated from the $\mathrm{RR}$ beat durations

\begin{tabular}{cccccccc}
$\begin{array}{c}\text { Subject } \\
\text { Number }\end{array}$ & hsEntropy & $(\mathbf{1}-$ hsDFA) & $\begin{array}{c}\text { Spectral } \\
\text { MTM }\end{array}$ & $\begin{array}{c}\text { Shannon } \\
\text { Entropy }\end{array}$ & ApEn & $\begin{array}{c}\text { Standard } \\
\text { Deviation }\end{array}$ & $\begin{array}{c}\text { Sample } \\
\text { Entropy }\end{array}$ \\
\hline $\mathbf{1}$ & -0.00582 & 0.00456 & 0.00518 & 0.00559 & 0.02590 & 0.01053 & 0.01738 \\
$\mathbf{2}$ & 0.00051 & -0.00038 & -0.00024 & -0.00026 & 0.00259 & 0.00592 & 0.00507 \\
$\mathbf{3}$ & -0.00437 & 0.00429 & 0.00690 & 0.00679 & 0.03016 & 0.01625 & 0.01476 \\
$\mathbf{4}$ & -0.00684 & 0.00605 & 0.00817 & 0.00801 & 0.03009 & 0.02147 & 0.02291 \\
$\mathbf{5}$ & -0.00079 & 0.00087 & 0.00144 & 0.00133 & 0.02362 & 0.01539 & 0.00847 \\
$\mathbf{6}$ & -0.00732 & 0.00665 & 0.00933 & 0.00919 & 0.02719 & 0.02142 & 0.01709 \\
$\mathbf{7}$ & -0.00548 & 0.00469 & 0.00548 & 0.00511 & 0.03813 & 0.02387 & 0.02065 \\
$\mathbf{8}$ & 0.00650 & -0.00375 & -0.00207 & -0.00251 & 0.01669 & 0.00734 & 0.01115 \\
$\mathbf{9}$ & -0.00521 & 0.00486 & 0.00657 & 0.00655 & 0.02298 & 0.03289 & 0.02738 \\
$\mathbf{1 0}$ & -0.00481 & 0.00436 & 0.00594 & 0.00615 & 0.02954 & 0.02314 & 0.01977 \\
$\mathbf{1 1}$ & -0.00408 & 0.00379 & 0.00559 & 0.00560 & 0.02345 & 0.01500 & 0.01180 \\
All & -0.00186 & 0.00202 & 0.00339 & 0.00330 & 0.02288 & 0.01411 & 0.01311 \\
\hline
\end{tabular}

Table 3: y-intercepts (Linear Regression) - Seven parameters versus gestational age for each individual fetus and then, for the complete dataset. The total number of recordings was $N=215$. The parameters were applied to the RR beat durations

\begin{tabular}{|c|c|c|c|c|c|c|c|}
\hline $\begin{array}{l}\text { Subject } \\
\text { Number }\end{array}$ & hsEntropy & (1-hsDFA) & $\begin{array}{l}\text { Spectral } \\
\text { MTM }\end{array}$ & $\begin{array}{c}\text { Shannon } \\
\text { Entropy }\end{array}$ & ApEn & $\begin{array}{l}\text { Standard } \\
\text { Deviation }\end{array}$ & $\begin{array}{l}\text { Sample } \\
\text { Entropy }\end{array}$ \\
\hline 1 & 0.5902 & 0.2121 & 0.5284 & 1.1070 & -0.3909 & 0.0493 & 0.0711 \\
\hline 2 & 0.3755 & 0.3773 & 0.7071 & 1.3010 & 0.2140 & 0.1591 & 0.3761 \\
\hline 3 & 0.4957 & 0.2640 & 0.5381 & 1.1330 & -0.4874 & -0.1238 & 0.1327 \\
\hline 4 & 0.5212 & 0.2490 & 0.5392 & 1.1390 & -0.5153 & -0.2594 & -0.0905 \\
\hline 5 & 0.3006 & 0.4382 & 0.7859 & 1.3870 & -0.2685 & -0.0604 & 0.3436 \\
\hline 6 & 0.5226 & 0.2451 & 0.5237 & 1.1190 & -0.3409 & -0.0537 & 0.1995 \\
\hline 7 & 0.5154 & 0.2571 & 0.5723 & 1.1790 & -0.7585 & -0.3718 & 0.0090 \\
\hline 8 & 0.3472 & 0.3731 & 0.6673 & 1.2640 & -0.1782 & 0.0698 & 0.1656 \\
\hline 9 & 0.4559 & 0.2989 & 0.6008 & 1.1980 & -0.3123 & -0.5089 & -0.0934 \\
\hline 10 & 0.4407 & 0.3165 & 0.6254 & 1.2180 & -0.3521 & -0.1527 & 0.2033 \\
\hline 11 & 0.4544 & 0.3038 & 0.5998 & 1.1940 & -0.3175 & -0.0490 & 0.2452 \\
\hline All & 0.4135 & 0.3374 & 0.6447 & 1.2410 & -0.2918 & -0.0254 & 0.2159 \\
\hline
\end{tabular}


Table 4: $\mathrm{R}^{2}$ values (Linear Regression) - The descriptive statistics for the seven parameters versus gestational age derived from each individual fetus. The number of subjects was eleven $(N=11)$. The parameters were applied to the RR beat durations

\begin{tabular}{|c|c|c|c|c|c|c|c|}
\hline $\begin{array}{l}\text { Prameter } \\
n=11\end{array}$ & hsEntropy & (1-hsDFA) & $\begin{array}{l}\text { Spectral } \\
\text { MTM }\end{array}$ & $\begin{array}{l}\text { Shannon } \\
\text { Entropy }\end{array}$ & ApEn & $\begin{array}{l}\text { Standard } \\
\text { Deviation }\end{array}$ & $\begin{array}{l}\text { Sample } \\
\text { Entropy }\end{array}$ \\
\hline Minimum & 0.007 & 0.006 & 0.002 & 0.002 & 0.034 & 0.100 & 0.063 \\
\hline 5\%Q & 0.011 & 0.013 & 0.013 & 0.012 & 0.161 & 0.129 & 0.115 \\
\hline $25 \% Q$ & 0.080 & 0.091 & 0.103 & 0.100 & 0.412 & 0.298 & 0.408 \\
\hline Median & 0.233 & 0.236 & 0.240 & 0.230 & 0.631 & 0.547 & 0.532 \\
\hline 75\%Q & 0.381 & 0.377 & 0.332 & 0.349 & 0.664 & 0.612 & 0.612 \\
\hline 95\%Q & 0.452 & 0.466 & 0.463 & 0.431 & 0.718 & 0.747 & 0.784 \\
\hline Maximum & 0.453 & 0.477 & 0.483 & 0.478 & 0.748 & 0.764 & 0.793 \\
\hline Range & 0.446 & 0.471 & 0.481 & 0.476 & 0.714 & 0.665 & 0.730 \\
\hline $90 \% \mathrm{Cl}$ & 0.441 & 0.453 & 0.450 & 0.419 & 0.557 & 0.617 & 0.669 \\
\hline IQRange & 0.301 & 0.287 & 0.229 & 0.249 & 0.252 & 0.314 & 0.204 \\
\hline
\end{tabular}

Regarding 'goodness-of-fit' evaluated by the R2 values for linear regression, ApEn measurement was optimum (Figure 3). The second best was SD, followed by SampEn with the remaining four measurement methods broadly having the same significance. In particular, the chaotic global R2 values are very similar (Figure 3 ). Table 4 examines the lower quartile $(25 \% \mathrm{Q})$, upper quartile (75\%Q) and inter-quartile ranges amongst other derivatives from the R2 data. This illustrates that ApEn has the highest median $\mathrm{R}^{2}$ value $(0.631)$ and one of the lowest inter-quartile ranges (Table 4). All median values (Table 4) were greater than the pooled datasets (ALL) in Table 1. Observing the values in Table 2 and Figure 4 for the slopes it is clear to see that the ApEn, SampEn and SD are the only parameters where the slopes are all positive for both the individual fetuses and the whole group combined. The other four measures include at least one negative slope. From Figure 5 and Table 3 on the y-intercepts of the linear regression we can observe that there is considerable variation across measurements but not so much within the measurements.

\section{DISCUSSION}

Previous studies by Porta et $\mathrm{al}^{39}$ and some of $u^{2,40-42}$ have investigated the relationship between linear analysis of fetal HRV and gestational age. All of these studies illustrated that HRV quantified using various measures is dependent on fetal age. Further investigations have included novel calculations on very short FMCG $^{43}$ and, power spectral analysis during periods of active and quite sleep during near and post term gestation. ${ }^{44}$

In this present study 'goodness-of-fit' $\left(\mathrm{R}^{2}\right)$ indicates how robustly the measures are associated with gestational age. For all measures, the individual $\mathrm{R}^{2}$ values are mostly higher than the values calculated over the pooled data. This corresponds to the results of our previous study and confirms that there is an individual component in the origin of fetal HRV. The $\mathrm{R}^{2}$ values for hsEntropy, hsDFA, sMTM and Shannon Entropy demonstrated a weak relationship to gestational age. Over all the subjects, on the basis of $\mathrm{R}^{2}$, gestational age generally explains less than $40 \%$ of the variation found in the individuals' values. For
SD, ApEn and SampEn, the explanatory power of gestational age is higher, and based on median $\mathrm{R}^{2}$ values more than $60 \%$ of the variation in the measurements can be explained by gestational age.

Of the measures that show good explanatory power, ApEn appears to have the highest, with generally higher $\mathrm{R}^{2}$ values as well as a lower spread of the values, particularly in the upper two quartiles. SampEn performs more consistently than SD, with slightly higher values and a smaller inter-quartile interval. This would suggest that these nonlinear measures are better at characterizing the relationship between individual HRV and gestational age than SD. The former quantify short-term HRV and take the order of the heartbeats into account whereas SD quantifies overall variance and is completely independent of the order in the data.

Slope quantifies the amount of change in the measures with respect to a unit of gestational age. The higher the slope, the more the value of the measure will change over time (i.e. gestational age). Table 2 illustrates that for all measures, the individual slopes are mostly higher than the slopes calculated over the pooled data. This suggests that there is an individual component in the origin of fetal HRV which is weakened when data is pooled.

The results here are similar to those for $\mathrm{R}^{2}$ : hsEntropy, hsDFA, sMTM and Shannon Entropy have relative low slopes (i.e. the values of these measures do not change much over time) whereas SD, ApEn and SampEn have higher slopes. Combined with the 'goodness-of-fit' results, this indicates that the latter 3 measures more consistently and clearly quantify the changes over gestational age and would be more useful in characterizing the maturation of the fetuses.

Of the 3 measures with high slopes, ApEn not only has the highest slopes, the values of the individual slopes are very similar: except for one individual (Subject 2), the values of the slopes are practically the same (i.e. the regression lines are almost parallel). The SampEn and SD slopes are somewhat lower and cover very similar ranges. They also vary more than those of ApEn. It seems that, of all the measures examined here, ApEn is the most consistent $\left(\mathrm{R}^{2}\right)$, the most distinct (slope value) and the most reliable (similarity of slopes between subjects) quantifier with respect to characterizing the change of HRV during pregnancy. 
The $y$-intercept gives us the value of the HRV measure at $\mathrm{x}=0$, which, in the context of pregnancy, means gestational age of zero, i.e. the time of conception. Its value is thus meaningless with respect to fetal HRV. However, if the slopes of several regression lines are the same, i.e. they are parallel, then the spread of the y-intercept values will quantify the distance between the lines. In the context of a fetal HRV measure, they will quantify how far apart the single fetuses are with respect to this measure. So, it only makes sense to look at the intercept if the slope values are very similar.

The slopes of hsEntropy, hsDFA, sMTM and Shannon Entropy are indeed similar; they have lower IQ-ranges than SampEn and SD (See Figure 4). Of these measures, the only informative one is ApEn (as mentioned previously). So it only really makes sense to take the y-intercept of ApEn into account.

It appears that, for any individual fetus, ApEn may be characterized by its y-intercept as the slope will be more or less be somewhere around 0.026 (based on our data). If one wanted to be less confounded by the slight differences between individual slopes (these differences will be exacerbated the farther you get away from the age range which is sensible), one could substitute the y-intercept value (at $\mathrm{x}=0$ ) by a physiologically meaningful value (e.g. at $\mathrm{x}=30$ weeks gestational age, corresponding to ApEn 0.45).

The newly introduced spectral nonlinear measures did not perform as well as those applied to time-series and RR beat durations, SD, ApEn and SampEn. These power spectra performed well when calculating the values of short time-series and comparing like with like. However, they failed to perform well when comparing their changes over time such as with gestational age. The reason proposed being that the power spectra are not as responsive to change as are time-series signals. The spectral values involved the heavy normalization of data and this reduced their responsiveness to variation. Only if the datasets are very short is the advantage of spectra beneficial. The important issue is the trade-off between the sensitivity of the parameters over time (gestational age), and the responsiveness to change over time-series. Shannon entropy is a non-spectral technique and appears to be deficient on both criterions.

Usually, changes in the HRV patterns are an indicator of health status. Higher HRV is generally a signal of good adaptation and characterize a healthy person with efficient autonomic mechanisms. On the other hand, lower $\mathrm{HRV}$ is frequently an indicator of insufficient ability of the autonomic nervous system to adapt to physiological or pathological challenges.

It is widely accepted that the complexity of the fetus HRV increases as the pregnancy progresses. Here most fetuses were examined for a period of at least 20 weeks, and the median time between data acquisitions was 1 week. Nevertheless, for the pooled dataset with all fetuses the strength of the relationship between measure and age was reduced, thus suggesting that the course of HRV is more consistent within individual fetuses over gestational age. In the cases where the slopes are all positive then we can deduce that the complexity of fetal HRV increases with gestational age. This is not the case for the four parameters which have a mixture of positive and negative slopes. The y-intercept is important because it informs us of the level of each complexity measurement. Across all measurements there is considerable variability (inter-individual), but within the values (intra-individual) there is not so much fluctuation.

The explanatory powers of the models we applied differed substantially between the seven fetal HRV measures studied. From the previous study ApEn and SD had the highest 'goodness-of-fit' values. SD which largely relates to overall variance contained in each time-series is the second best with the HRV of some individuals captured poorly by the model and that of other individuals favourably. Statistically, it is clear that many of the other complexity measures based on spectral nonlinear dynamics are unable to exceed the significance of ApEn. Although between ApEn and SampEn there is no significant difference. Nevertheless, as mentioned in Correlation - Least Squares Method section reduced SampEn indicates greater self-similarity than similar values for ApEn.

It is also important to consider why the chaotic global values perform so badly and unable to outperform ApEn, SampEn and SD. We suggest that the basis for this is that they are focussed on power spectra alone. It is unlikely to be due to noise or the length of the datasets since they were specifically designed to overcome these potential pitfalls. They have been proven on many situations such as diabetes, ${ }^{16}$ childhood obesity ${ }^{23}$ and Chronic Obstructive Pulmonary Disease. ${ }^{17}$ There are numerous other techniques which could be employed such as fractal analysis, ${ }^{45}$ a few other entropies such as Renyi ${ }^{20}$ and Tsallis ${ }^{37}$ amongst others.

A further limitation of this study is that there is a relatively small number of subjects in the dataset. Additionally, there is an uneven distribution of measurements both with respect to gestational age and the number of acquisitions per subject. Correspondingly with regards to the chaotic global parameters, ${ }^{12}$ they are only presented as single entities whereas in other studies ${ }^{11,46,47}$ there have been seven different permutations of the three chaotic global techniques which we refer to as chaos forward parameters (CFP1 to CFP7).

As the result of the study was negative it is difficult to propose any clinical benefit except that it reinforces the use of ApEn and SampEn as techniques to be applied in such situations. Nonetheless, fetal HRV generally, has been almost invariably been justified by potential clinical use. This is covered extensively in the papers by Hoyer et $\mathrm{al}^{4,48,49}$.

In conclusion ApEn remains the optimum measure of complexity for those examined here during these situa $\neg$ tions of fetal development. The next best are SampEn and SD. Nevertheless, the three "chaotic global" spectrally adapted parameters have higher statistical significance than the Shannon Entropy. It is proposed that there is a trade-off between responsiveness to short time-series where the spectrally derived parameters excel with the responsiveness over time (gestational age) where the ApEn and SampEn perform best because there is less normalization and more fluctuation during the time-series as- 
sessment. Additionally, fetal HRV has an individual aspect which is weakened when data is pooled -- individual slopes are higher than the slopes calculated over the pooled data.

\section{ACKNOWLEDGEMENTS}

The authors declare that there is no conflict of interests regarding the publication of this article.

\section{REFERENCES}

1. Van Leeuwen P, Cysarz D, Edelhauser F, Gronemeyer D. Heart rate variability in the individual fetus. Auton Neurosc. 2013;178(1-2):24-8. DOI: http://dx.doi.org/10.1016/j.autneu.2013.01.005

2. Van Leeuwen P, Lange S, Bettermann H, Gronemeyer D, Hatzmann W. Fetal heart rate variability and complexity in the course of pregnancy. Early Hum Dev. 1999;54(3):259-69. DOI: http://dx.doi.org/10.1016/ S0378-3782(98)00102-9

3. Gieraltowski J, Hoyer D, Tetschke F, Nowack S, Schneider U, Zebrowski J. Development of multiscale complexity and multifractality of fetal heart rate variability. Auton Neurosc. 2013;178(1-2):29-36. DOI: http://dx.doi.org/10.1016/j.autneu.2013.01.009

4. Hoyer D, Nowack S, Bauer S, Tetschke F, Rudolph A, Wallwitz U, et al. Fetal development of complex autonomic control evaluated from multiscale heart rate patterns. Am J Physiol Regul Integr Comp Physiol. 2013;304(5):R383-92. DOI: http://dx.doi.org/10.1152/ajpregu.00120.2012

5. Billman GE. Heart rate variability - a historical perspective. Front Physiol. 2011;2:86. DOI: http://dx.doi. org/10.3389/fphys.2011.00086

6. Schneider U, Schleussner E, Fiedler A, Jaekel S, Liehr M, Haueisen J, et al. Fetal heart rate variability reveals differential dynamics in the intrauterine development of the sympathetic and parasympathetic branches of the autonomic nervous system. Physiol Meas. 2009;30(2):215-26. DOI: http://dx.doi. org/10.1088/0967-3334/30/2/008

7. Romanini C, Rizzo G. Fetal behaviour in normal and compromised fetuses. An overview. Early Hum Dev. 1995;43(2):117-31.

8. Lange S, Van Leeuwen P, Schneider U, Frank B, Hoyer D, Geue D, et al. Heart rate features in fetal behavioural states. Early Hum Dev. 2009;85(2):131-5. DOI: http://dx.doi.org/10.1016/j.earlhumdev.2008.07.004

9. Gonçalves H, Bernardes J, Rocha AP, Ayres-de-Campos D. Linear and nonlinear analysis of heart rate patterns associated with fetal behavioral states in the antepartum period. Early Hum Dev. 2007;83(9):58591. DOI: http://dx.doi.org/10.1016/j.earlhumdev.2006.12.006

10. Pincus SM, Goldberger AL. Physiological time-series analysis: what does regularity quantify? Am J Physiol. 1994;266(4 Pt 2):H1643-56.

11. Barreto GS, Vanderlei FM, Vanderlei LCM, Garner DM. Risk appraisal by novel chaotic globals to HRV in subjects with malnutrition. J Hum Growth Dev. 2014;24(3):243-8. DOI: http://dx.doi.org/10.7322/ jhgd.88900

12. Garner DM, Ling BWK. Measuring and locating zones of chaos and irregularity. J Syst Sci Complex. 2014;27(3):494-506. DOI: http://dx.doi.org/10.1007/s11424-014-2197-7

13. Shannon CE. A mathematical theory of communication. ACM Sigmobile Mobile Comp Communic Rev. 2001;5(1):3-55. DOI: http://dx.doi.org/10.1145/584091.584093

14. Peng CK, Havlin S, Stanley HE, Goldberger AL. Quantification of scaling exponents and crossover phenomena in nonstationary heartbeat time series. Chaos. 1995;5(1):82-7. DOI: http://dx.doi. org/10.1063/1.166141

15. Antonio AMS, Garner DM, Cardoso MA, Abreu LC, Raimundo RD, Navega MT, et al. Behaviour of globally chaotic parameters of heart rate variability following a protocol of exercise with flexible pole. Russ J Cardiol. 2015;4(120):24-8. DOI: http://dx.doi.org/10.15829/1560-4071-2015-04-22-26

16. Souza NM, Vanderlei LCM, Garner DM. Risk evaluation of diabetes mellitus by relation of chaotic globals to HRV. Complexity. 2015;20(3):84-92. DOI: http://dx.doi.org/10.1002/cplx.21508

17. Bernardo AFB, Vanderlei LCM, Garner DM. HRV analysis: a clinical and diagnostic tool in chronic obstructive pulmonary disease. Int Schol Res Notices. 2014;2014:6. DOI: http://dx.doi.org/10.1155/2014/673232

18. Richman JS, Moorman JR. Physiological time-series analysis using approximate entropy and sample entropy. Am J Physiol Heart Circ Physiol. 2000;278(6):H2039-49.

19. Camm AJ, Malik M, Bigger JT, Breithardt G, Cerutti S, Cohen RJ, et al. Heart rate variability: standards of measurement, physiological interpretation and clinical use. Task Force of the European Society of Cardiology and the North American Society of Pacing and Electrophysiology. Circulation. 1996;93(5):1043-65. DOI: http://dx.doi.org/10.1161/01.CIR.93.5.1043

20. Zyczkowski K. Renyi extrapolation of Shannon entropy. Open Syst Inf Dyn. 2003;10(3):297-310. DOI: http://dx.doi.org/10.1023/A:1025128024427 
21. Ryabko B, Reznikova Z. Using Shannon entropy and Kolmogorov complexity to study the communicative system and cognitive capacities in ants. Complexity. 1996;2(2):37-42. DOI: 10.1002/(SICI)10990526(199611/12)

22. Ho MW. The rainbow and the worm: The physics of organisms. 3rd ed. Singapore: World scientific; 2008.

23. Vanderlei FM, Vanderlei LCM, Garner DM. Chaotic global parameters correlation with heart rate variability in obese children. J Hum Growth Dev. 2014;24(1):24-30. DOI: http://dx.doi.org/10.7322/jhgd.72041

24. Percival DB, Walden AT. Spectral analysis for physical applications: multitaper and conventional univariate techniques. New York: Cambridge University Press; 1993.

25. Alkan A, Kiymik MK. Comparison of AR and Welch methods in epileptic seizure detection. J Med Sys. 2006;30(6):413-9. DOI: http://dx.doi.org/10.1007/s10916-005-9001-0

26. Ghil M. The SSA-MTM Toolkit: applications to analysis and prediction of time series. Proc SPIE. 1997;3165:216-30. DOI: http://dx.doi.org/10.1117/12.279594

27. Slepian D. Prolate spheroidal wave functions, Fourier analysis and uncertainty, V: the discrete case. Bell Syst Tech J. 1978;57(5):1371-430. DOI: http://dx.doi.org/10.1002/j.1538-7305.1978.tb02104.x

28. Gould SH. Variational methods for eigenvalue problems: an introduction to the methods of Rayleigh, Ritz, Weinstein, and Aronszajn. Courier Dover Publications; 1995.

29. Johnson R, Shore J. Which is the better entropy expression for speech processing:-S log $\mathrm{S}$ or log $\mathrm{S}$ ? IEEE Transact Acoustics, Speech, Signal Proc. 1984;32(1):129-37. DOI: http://dx.doi.org/10.1109/ TASSP.1984.1164296

30. Peng CK, Havlin S, Hausdorff JM, Mietus JE, Stanley HE, Goldberger AL. Fractal mechanisms and heart rate dynamics. Long-range correlations and their breakdown with disease. J Electrocardiol. 1995;28(Suppl. 1):59-65. DOI: http://dx.doi.org/10.1016/S0022-0736(95)80017-4

31. Donaldson GC, Seemungal TA, Hurst JR, Wedzicha JA. Detrended fluctuation analysis of peak expiratory flow and exacerbation frequency in COPD. Eur Respir J. 2012;40(5):1123-9. DOI: http://dx.doi. org/10.1183/09031936.00180811

32. Mann ME, Lees JM. Robust estimation of background noise and signal detection in climatic time series. Climatic Change. 1996;33(3):409-45. DOI: http://dx.doi.org/10.1007/BF00142586

33. Thomson DJ. Spectrum estimation and harmonic analysis. Proc IEEE. 1982;70(9):1055-96. DOI: http:// dx.doi.org/10.1109/PROC.1982.12433

34. Batista AM, Viana RL. Kolmogorov-Sinai entropy for locally coupled piecewise linear maps. Physica A: Stat Mechan Applicat. 2002;308(1-4):125-34. DOI: http://dx.doi.org/10.1016/S0378-4371(02)00579-4

35. Hornero R, Abasolo D, Escudero J, Gomez C. Nonlinear analysis of electroencephalogram and magnetoencephalogram recordings in patients with Alzheimer's disease. Philos Trans A Math Phys Eng Sci. 2009;367(1887):317-36. DOI: http://dx.doi.org/10.1098/rsta.2008.0197

36. Richman JS, Lake DE, Moorman JR. Sample entropy. Methods Enzymol. 2004;384:172-84. DOI: http:// dx.doi.org/10.1016/S0076-6879(04)84011-4

37. Silva LEV, LO Murta Jr. Evaluation of physiologic complexity in time series using generalized sample entropy and surrogate data analysis. Chaos. 2012;22(4):043105. DOI: http://dx.doi.org/10.1063/1.4758815

38. Ramdani S, Bouchara F, Lagarde J. Influence of noise on the sample entropy algorithm. Chaos. 2009;19(1):013123. DOI: http://dx.doi.org/10.1063/1.3081406

39. Porta A, Casali KR, Casali AG, Gnecchi-Ruscone T, Tobaldini E, Montano N, et al. Temporal asymmetries of short-term heart period variability are linked to autonomic regulation. Am J Physiol Regul Integr Comp Physiol. 2008;295(2):R550-7. DOI: http://dx.doi.org/10.1152/ajpregu.00129.2008

40. Van Leeuwen P, Geue D, Lange S, Hatzmann W, Grönemeyer D. Changes in the frequency power spectrum of fetal heart rate in the course of pregnancy. Prenat Diagn. 2003;23(11):909-16. DOI: http://dx.doi. org/10.1002/pd.723

41. Lange S, Van Leeuwen P, Geue D, Hatzmann W, Gronemeyer D. Influence of gestational age, heart rate, gender and time of day on fetal heart rate variability. Med Biol Eng Comput. 2005;43(4):481-6. DOI: http://dx.doi.org/10.1007/BF02344729

42. Van Leeuwen P, Lange S, Geue D, Gronemeyer D. Heart rate variability in the fetus: a comparison of measures. Biomed Tech (Berl). 2007;52(1):61-5. DOI: http://dx.doi.org/10.1515/BMT.2007.012

43. Moraes ER, Murta LO, Baffa O, Wakai RT, Comani S. Linear and nonlinear measures of fetal heart rate patterns evaluated on very short fetal magnetocardiograms. Physiol Meas. 2012;33(10):1563-83. DOI: http://dx.doi.org/10.1088/0967-3334/33/10/1563

44. van Laar J, Peters C, Vullings R, Houterman S, Oei SG. Power spectrum analysis of fetal heart rate variability at near term and post term gestation during active sleep and quiet sleep. Early Hum Dev. 2009;85(12):795-8. DOI: http://dx.doi.org/10.1016/j.earlhumdev.2009.11.001

45. Anier A, Lipping T, Melto S, Hovilehto S. Higuchi fractal dimension and spectral entropy as measures of depth of sedation in intensive care unit. Conf Procl EEE Eng Med Biol Soc. 2004;1:526-9. DOI: http:// dx.doi.org/10.1109/IEMBS.2004.1403210 
46. Vanderlei FM, Vanderlei LCM, Garner DM. Heart rate dynamics by novel chaotic globals to HRV in obese youths. J Hum Growth Dev. 2015;25(1):82-8. DOI: http://dx.doi.org/10.7322/jhgd.96772

47. Wajnsztejn R, De Carvalho TD, Garner DM, Vanderlei LCM, Godoy MF, Raimundo RD, et al. Heart rate variability analysis by chaotic global techniques in children with attention deficit hyperactivity disorder. Complexity. 2016:21(6): 412-19. DOI: http://dx.doi.org/10.1002/cplx.21700

48. Hoyer D, Kowalski EM, Schmidt A, Tetschke F, Nowack S, Rudolph A, et al. Fetal autonomic brain age scores, segmented heart rate variability analysis, and traditional short term variability. Front Hum Neurosci. 2014; 8:948. DOI: http://dx.doi.org/10.3389/fnhum.2014.00948

49. Hoyer D, Tetschke F, Jaekel S, Nowack S, Witte OW, Schleussner E, et al. Fetal functional brain age assessed from universal developmental indices obtained from neuro-vegetative activity patterns. PLoS One. 2013;8(9): e74431. DOI: http://dx.doi.org/10.1371/journal.pone.0074431

This article is distributed under the terms of the Creative Commons Attribution 4.0 International License (http://creativecommons.org/licenses/by/4.0/), which permits unrestricted use, distribution, and reproduction in any medium, provided you give appropriate credit to the original author(s) and the source, provide a link to the Creative Commons license, and indicate if changes were made. The Creative Commons Public Domain Dedication waiver (http://creativecommons.org/publicdomain/zero/1.0/) applies to the data made available in this article, unless otherwise stated.

\section{Resumo}

Introdução: A freqüência cardíaca fetal e da sua variabilidade durante o curso da gestação têm sido extensivamente pesquisada. A redução global da frequência cardíaca e aumento da VFC fetal está associada com o crescimento fetal e aumento da integração neural. O aumento da complexidade das exigências sobre o sistema cardiovascular conduz a uma maior variação no decurso temporal da frequência cardíaca o que foi mostrado para reflectir-se medidas de complexidade. O objetivo deste trabalho foi investigar medidas de complexidade novos em relação à sua capacidade de quantificar as mudanças ao longo da idade gestacional em fetos individuais de forma consistente e de forma estável.

Método: Foram examinados 215 magnetocardiograms fetais (FMCG), cada um dos 5 min de duração, em 11 fetos durante o segundo e terceiro trimestres (pelo menos 10 conjuntos de dados por feto). A partir do grande consumo determinamos as durações RR batimento fetais. Para cada série temporal 5 min dos intervalos RR então calculada Shannon entropia, alta entropia espectral, alta espectral Destendenciada Análise Flutuação, espectral Multi-Taper Método, bem como o desvio padrão e duas medidas de complexidade comumente utilizados: aproximado Entropia e Amostra Entropia. Para cada medida assunto e HRV, foi realizada análise de regressão em relação à idade gestacional. O coeficiente de determinação $R^{2}$ foi usada para estimar a "o bem-of-fit", a inclinação da regressão indicou a força do indivíduo dependência da idade gestacional.

Resultados: Verificou-se que as novas medidas de complexidade não superar ApEn.

Conclusão: Este estudo agora rejeitou a hipótese de que as medidas de complexidade espectrais superar os aplicados anteriormente.

Palavras-chave: entropia, variabilidade da frequência cardíaca, complexidade, caos, poder espectros. 